\title{
Genome-wide association study for milk infrared wavenumbers
}

\author{
Qiuyu Wang and Henk Bovenhuis ${ }^{1}$ \\ Animal Breeding and Genomics Centre, Wageningen University, PO Box 338,6700AH, Wageningen, the Netherlands
}

\begin{abstract}
Individual wavenumbers of the infrared (IR) spectra of bovine milk have been shown to be moderately to highly heritable. The objective of this study was to identify genomic regions associated with individual milk IR wavenumbers. This is expected to provide information about the genetic background of milk composition and give insight in the relation between IR wavenumbers and milk components. For this purpose, a genome-wide association study was performed for a selected set of 50 individual IR wavenumbers measured on 1,748 Dutch Holstein cows. Significant associations were detected for 28 of the 50 wavenumbers. In total, 24 genomic regions distributed over 16 bovine chromosomes were identified. Major genomic regions associated with milk IR wavenumbers were identified on chromosomes 1,5 , $6,14,19$, and 20. Most of these regions also showed significant associations with fat, protein, or lactose percentage. However, we also identified some new regions that were not associated with any one of these routinely collected milk composition traits. On chromosome 1, we identified 2 new genomic regions and hypothesized that they are related to variation in milk phosphorus content and orotic acid, respectively. On chromosome 20, we identified a new genomic region that seems to be related to citric acid. Identification of genomic regions associated with milk phosphorus content, orotic acid, and citric acid suggest that the milk IR spectra contain direct information on these milk components. Consequently milk IR analyses probably can be used to predict these milk components, which have low concentrations in milk; this can lead to novel applications of milk IR spectroscopy for dairy cattle breeding and herd management.
\end{abstract}

Key words: bovine milk, infrared wavenumbers, genome-wide association study

Received July 7, 2017

Accepted November 20, 2017.

${ }^{1}$ Corresponding author: Henk.Bovenhuis@wur.nl

\section{INTRODUCTION}

Infrared (IR) spectroscopy is a fast and relatively cheap method to determine milk composition. It is the standard method for routine quantification of fat, protein, and lactose content of milk (ICAR, 2012). Several studies showed that IR can also be used to determine milk fat composition (e.g., Soyeurt et al., 2006; Rutten et al., 2009). More recently it has been suggested that milk IR spectra also can be used to predict other characteristics, such as negative energy balance and methane emission of dairy cows (McParland et al., 2011; Dehareng et al., 2012). The IR spectra are caused by the absorption of electromagnetic radiation at wavenumbers that are correlated with the vibrations (stretching and bending) of specific chemical bonds within a molecule (Sun, 2009). The analysis of milk IR applies mainly the region 400 to $4,000 \mathrm{~cm}^{-1}$ or 2,500 to $25,000 \mathrm{~nm}$ due to active vibrations of various chemical bonds in different small regions. By assigning absorption bands of chemical bonds in IR to milk components, we can identify milk IR wavenumbers associated with some common milk components, such as milk fat, milk protein, and lactose. The assignment is based on the fact that the major chemical bonds with known vibration frequencies are abundant in these common milk components. However, due to the complexity of milk, it is sometimes difficult to relate wavenumbers to specific components. Milk IR spectra result from a comprehensive scan of milk and may contain information on milk components that are currently not quantified.

Several genome-wide association studies (GWAS) have been performed for routinely recorded milk production traits such as milk yield, fat, and protein content (e.g., Daetwyler et al., 2008; Pryce et al., 2010; Cole et al., 2011); more recently some studies performed GWAS for detailed milk fat and protein composition (Bouwman et al., 2011; Schopen et al., 2011). Buitenhuis et al. (2013) performed a GWAS for milk components based on nuclear magnetic resonance spectroscopy. More recently Sanchez et al. (2016) performed a GWAS based on IR-predicted milk protein composition. The bovine milk IR spectrum might provide unique information about the genetic background of milk composition and, 
to our knowledge, no GWAS based on bovine milk IR wavenumbers has been reported previously.

Genetic analyses of bovine milk IR showed that most IR wavenumbers are heritable (e.g., Soyeurt et al., 2010; Bittante and Cecchinato, 2013). Wang et al. (2016) showed that the majority of the wavenumbers have moderate to high interherd heritabilities, ranging from 0.20 to 0.60 . Performing a GWAS for milk IR wavenumbers will provide new possibilities to identify genomic regions responsible for differences in milk composition and enhance our understanding of the background of bovine milk IR spectra. The objective of our study was to perform GWAS for a representative set of milk IR wavenumbers to unravel the genetic background of milk IR spectra and better understand the relationship between milk IR wavenumbers and milk components.

\section{MATERIALS AND METHODS}

\section{Data}

Data used for the current study was based on 1 morning milk sample from 1,748 first-parity HolsteinFriesian cows. Milk samples were collected from February to March 2005. All cows had at least $87.5 \%$ Holstein-Friesian genes. The population consisted of 5 large paternal half-sib families from proven sires (98 to 196 daughters per sire) and 50 small paternal half-sib families from test sires ( 8 to 23 daughters per sire), as well as 168 cows descending from 44 other proven sires ( 1 to 25 daughters per sire) to ensure at least 3 cows per herd. The pedigree of the cows was supplied by CRV (Cooperative cattle improvement organization, Arnhem, the Netherlands).

Milk IR spectra were recorded in a $10-\mathrm{mL}$ milk sample using the same MilkoScan FT 6000 equipment (FOSS, Hillerød, Denmark) at the certified laboratory of the Dutch Milk Control Station QLIP (Zutphen, the Netherlands). The spectra consist of the transmittance values measured at 1,060 individual wavenumbers between 925 and $5,008 \mathrm{~cm}^{-1}$. Transmittance quantifies the amount of light passing through the milk sample.

Phenotypic correlations among individual wavenumbers were analyzed using PROC CLUSTER in SAS 9.3 (SAS Institute, 2011). More than $95 \%$ of the phenotypic variance in the 1,060 wavenumbers could be explained based on 50 clusters. From each of these 50 clusters, 1 wavenumber was selected for the GWAS. If a cluster contained multiple consecutive wavenumbers, the middle wavenumber was selected. We also performed GWAS for fat, protein, and lactose percentage based on the same milk samples. Fat, protein, and lactose percentage of the same milk samples were predicted based on IR analyses by the Dutch Milk Control Station QLIP (Zutphen, the Netherlands).

\section{Genotypes}

Blood samples were collected from the cows for DNA isolation. A custom 50K SNP Infinium Array (Illumina, San Diego, CA) designed by CRV was used for genotyping. The cows were genotyped for 50,688 SNP. The SNP were mapped using the bovine genome assembly BTAU_4.0 (Liu et al., 2009). Among these SNP, 778 were not mapped to any of the Bos taurus chromosomes and were therefore assigned to BTA 0. In total, 591 SNP were assigned to the X chromosome.

\section{Statistical Analysis}

The association study for each IR wavenumber was performed based on the model

$$
\begin{aligned}
& y_{i j k l m n}=\mu+\beta_{1} \times \text { lactst }_{i j k l m n}+\beta_{2} \times \text { afc }_{i j k l m n}+\text { season }_{i} \\
& + \text { sirecode }_{j}+\text { date }_{k}+\text { SNP }_{l}+\text { herd }_{m}+a_{n}+e_{i j k l m n}, \quad[1]
\end{aligned}
$$

where $y_{i j k l m n}$ is the transmittance value of the IR wavenumber, or predicted milk fat, protein, and lactose percentage; $\mu$ is the general mean; lactst $t_{i j k l m n}$ is a covariate for the effect of lactation stage (63 to $282 \mathrm{~d}$ ) with regression coefficient $\beta_{1}$; af $c_{i j k l m n}$ is a covariate for the effect of age at first calving with regression coefficient $\beta_{2} ;$ season $_{i}$ is the fixed effect accounting for season of calving (June-August 2004, September-November 2004 or December 2004-January 2005); sirecode $_{j}$ is the fixed effect accounting for possible differences in genetic level between the groups of proven bull daughters and young bull daughters; date $e_{k}$ is the fixed effect accounting for the effect of day at which IR analyses of milk samples took place (k ranges from 1 to 17 ); $S N P_{l}$ is the fixed effect of SNP genotype; herd $_{m}$ is a random effect of herd $m$, distributed as $N\left(0, \mathbf{I} \sigma_{h}^{2}\right)$, with identity matrix $\mathbf{I}$ and herd variance $\sigma_{h}^{2} ; a_{n}$ is a random additive genetic effect of animal $n$, distributed as $N\left(0, \mathbf{A} \sigma_{a}^{2}\right)$, with additive genetic relationship matrix $\mathbf{A}$ and the additive genetic variance $\sigma_{a}^{2}$ (the additive genetic relationship matrix was constructed based on 12,548 animals); $e_{i j k l m n}$ is a random residual effect, distributed as $N\left(0, \mathbf{I} \sigma_{e}^{2}\right)$, with error variance $\sigma_{e}^{2}$.

In the GWAS variance components were fixed at values estimated and presented by Wang et al. (2016) using the same data (see Table 1). Analyses were performed using ASReml (Gilmour et al., 2009).

The genome-wide false discovery rate (FDR) was used to determine significance; FDR were determined 
Table 1. Descriptive statistics: estimates of proportion of variance due to herd and heritability, phenotypic standard deviation $\left(\sigma_{\mathrm{p}}\right)$, related chemical bonds, and the associated genomic regions of infrared wavenumbers and milk production traits in this study

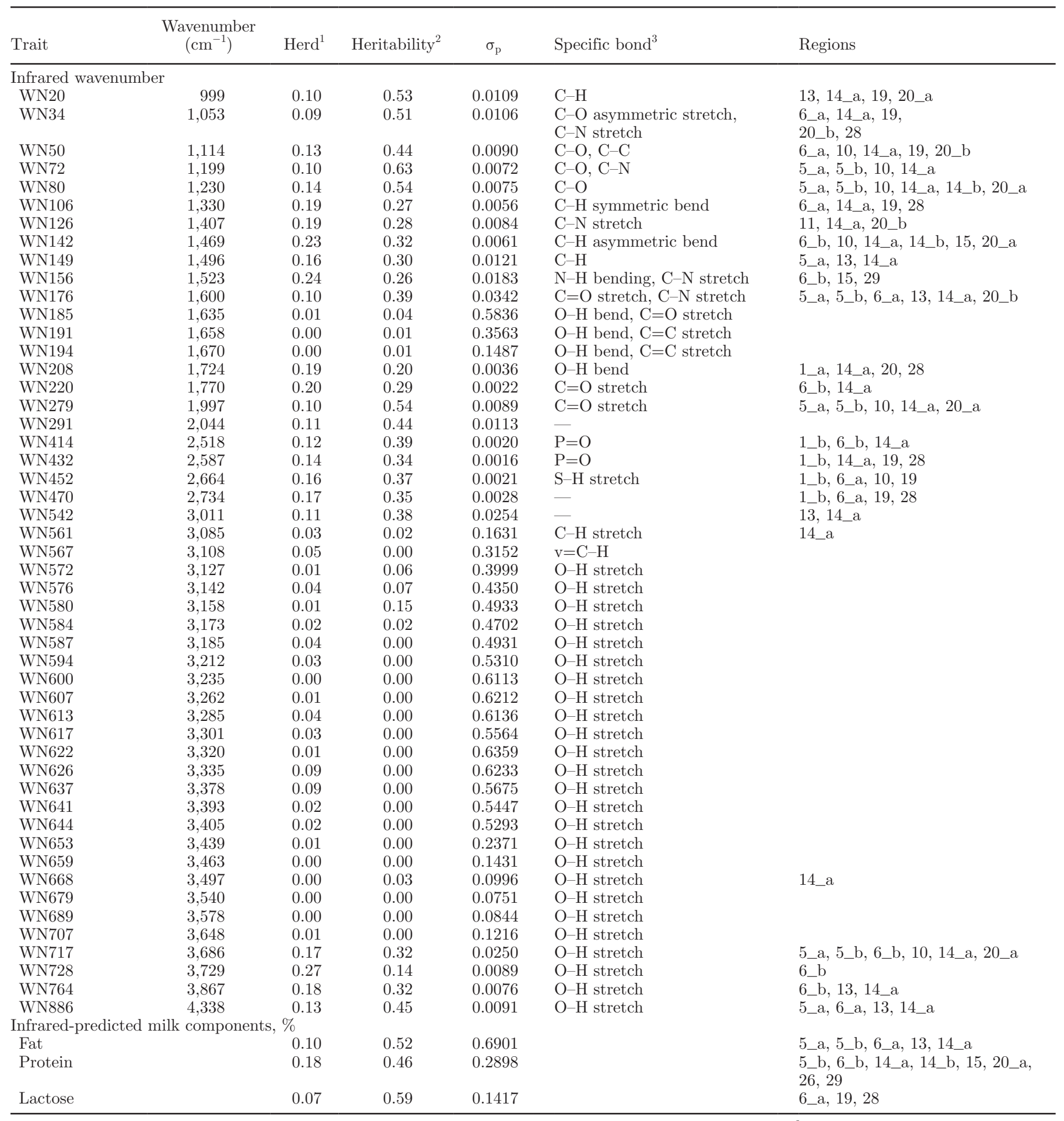

${ }^{1}$ Herd describes the proportion of total variance explained by the differences between herds. Herd $=\frac{\sigma_{h}^{2}}{\sigma_{a}^{2}+\sigma_{h}^{2}+\sigma_{e}^{2}}$, where $\sigma_{a}^{2}$ is additive genetic
variance, $\sigma_{h}^{2}$ is the variance due to differences between herds, $\sigma_{e}^{2}$ is the residual variance. ${ }^{2}$ Heritability $=\frac{\sigma_{a}^{2}}{\sigma_{a}^{2}+\sigma_{h}^{2}+\sigma_{e}^{2}}$, where variables are as described for herd.

${ }^{3}$ This is according to Sun (2009) and Diem (2015). 
for each trait separately using the R (R Core Team, 2013) package qvalue. Associations with a FDR $<0.01$ were considered significant. A sensitivity analysis was performed in case the lead SNP had a genotype class with at least 1 but less than 5 observations. In that case the records for the minor genotype class were excluded from the analysis and the analysis was repeated based on the remaining genotype classes.

In the current study, a genomic region containing at least $2 \mathrm{SNP}$ located within $10 \mathrm{Mbp}$ with a significant effect on a trait is defined as a significant region for that trait. The region extends until the last significant SNP was not followed by another significant SNP within 10 Mbp.

\section{RESULTS AND DISCUSSION}

Table 1 shows the IR wavenumbers that were selected for the current study and IR-predicted fat, protein, and lactose percentage. Table 1 shows the proportion of the variance due to herd and genetics for each of the wavenumbers and information regarding chemical bonds with specific absorption bands for the selected wavenumbers (Sun, 2009; Diem, 2015). Several of the selected wavenumbers were located in the water absorption region (between 1,619 and 1,674 and between 3,073 and $\left.3,667 \mathrm{~cm}^{-1}\right)$. These wavenumbers were included in the GWAS as a result of the applied wavenumber selection procedure (i.e., these wavenumbers tend to have low correlations with other wavenumbers). Some of the selected wavenumbers are important for the quantification of milk fat, protein, and lactose percentage due to the absorptions of abundant chemical bonds in these molecules.

Correlations among wavenumbers analyzed in this study, including IR-predicted fat, protein, and lactose percentage are graphically illustrated using a cluster tree (Figure 1). Wavenumbers from the water absorption region (e.g., WN587, WN626, and WN641) show weak correlations with other groups of wavenumbers. Many of the selected wavenumbers are related to IR-predicted fat, protein, or lactose percentage. Some of them show especially strong correlations with fat (WN542), protein (WN717), or lactose percentage (WN34).

The significant genomic regions detected in the GWAS are summarized in Table 1, and genome-wide association plots for fat, protein, and lactose percentage are shown in Figure 2. For the IR wavenumbers, not all genome-wide association plots are shown; Figure 3 shows plots for those wavenumbers that show a strong signal in some of the chromosomal regions. The y-axis in Figure 2 and 3 are cut of at a $-\log _{10}(P)$ value of 20 .

The GWAS results indicated that 28 out of the 50 wavenumbers studied were significantly associated with at least 1 genomic region. Significant associations were detected for in total 24 genomic regions distributed over 16 bovine chromosomes. No genomic regions were associated with all selected wavenumbers. Chromosomes BTA 1, BTA 5, BTA 6, BTA 14, BTA 19, and BTA 20 showed significant associations with multiple wavenumbers or multiple regions on those chromosomes showed significant associations. Results from these chromosomes will be discussed in more detail.

\section{BTA 1}

We detected 2 significant regions on BTA 1: region 1_a, from 56.9 to 80.8 Mbp (see Figure 3, WN208), and region 1_b, from 145.4 to 147.3 Mbp (see Figure 3, WN432). These 2 regions did not show significant associations in our study with the routinely recorded milk composition traits fat, protein, and lactose percentage (Figure 2), and to our knowledge these regions also have not been reported to be associated with routinely recorded milk production traits in other studies. Region 1_a is associated with WN208 and the lead SNP is rs29022932 with a $-\log _{10}(P)$ of 7.2 is located at 76.6 Mbp. Region 1_b showed significant associations for WN414, WN432, WN452, and WN470 with $-\log _{10}(P)$ ranging from 6.2 to 8.9. The SNP rs29019625 showed the strongest association for WN414, and an adjacent SNP rs43281569 showed the strongest association for WN432, WN452, and WN470.

Region 1_a, associated with WN208, harbors the gene uridine monophosphate synthase (UMPS), which catalyzes the last 2 steps of de novo pyrimidine synthesis converting orotic acid to $5^{\prime}$-monophosphate. This gene is known for the lethal genetic defect DUMPS (deficiency of uridine mono phosphate synthase). Female carriers of this defect are known to have elevated levels of orotic acid in their milk and urine (Robinson et al., 1984). Buitenhuis et al. (2013) reported a QTL for orotic acid in the same region as the current study detected. Buitenhuis et al. (2013) quantified orotic acid using nuclear magnetic resonance spectroscopy. Based on these results we hypothesized that milk IR spectra can be used to predict orotic acid in milk.

In region 1_b, located near $146 \mathrm{Mbp}$, we found several SNP (e.g., rs29019625) significantly associated with WN414, WN432, WN452, and WN470. Some of these wavenumbers are related to $\mathrm{P}=\mathrm{O}$ chemical bonds (Table 1). Using inductively coupled plasma emission spectroscopy, Buitenhuis et al. (2015) and Kemper et al. (2016) identified a QTL at 144.4 Mbp for milk phosphorus concentration. These studies suggested SLC37A1 (solute carrier family 37 member 1 ) as the most likely candidate gene, explaining approximately $10 \%$ phenotypic variance of phosphorus concentration. 
Gene SLC37A1 functions as a phosphorus antiporter (Chou et al., 2013), transporting glucose-6-phosphate and phosphorus in opposite directions.

It has been shown that milk phosphorus content can be predicted based on milk IR spectra (Soyeurt et al., 2009; Toffanin et al., 2015; Bonfatti et al., 2016). This prediction might be based on the relation between milk protein content and milk phosphorus content. However, region 1_b did not show an association with protein percentage in our study (Figure 2); therefore, these results suggest that the milk IR spectra contain direct information on milk phosphorus content. The current study provides additional evidence that milk phosphorus content can be predicted by milk IR spectra.
Large-scale quantification of milk phosphorus content using infrared analyses might result in management strategies to better feed cows according their phosphorus requirement and in more efficient use of phosphorus by the dairy sector.

\section{BTA 5}

We detected 2 significant regions on BTA 5: region 5_a, from 43.0 to $47.0 \mathrm{Mbp}$ (see Figure 2 for fat percentage and Figure 3 for WN149), and region $5 \_b$, from 97.4 to $101.1 \mathrm{Mbp}$ (see Figure 2 for fat and protein percentage and Figure 3 for WN80). Region 5_a contained 2 SNP (rs41616530 and rs29014575) showing signifi-

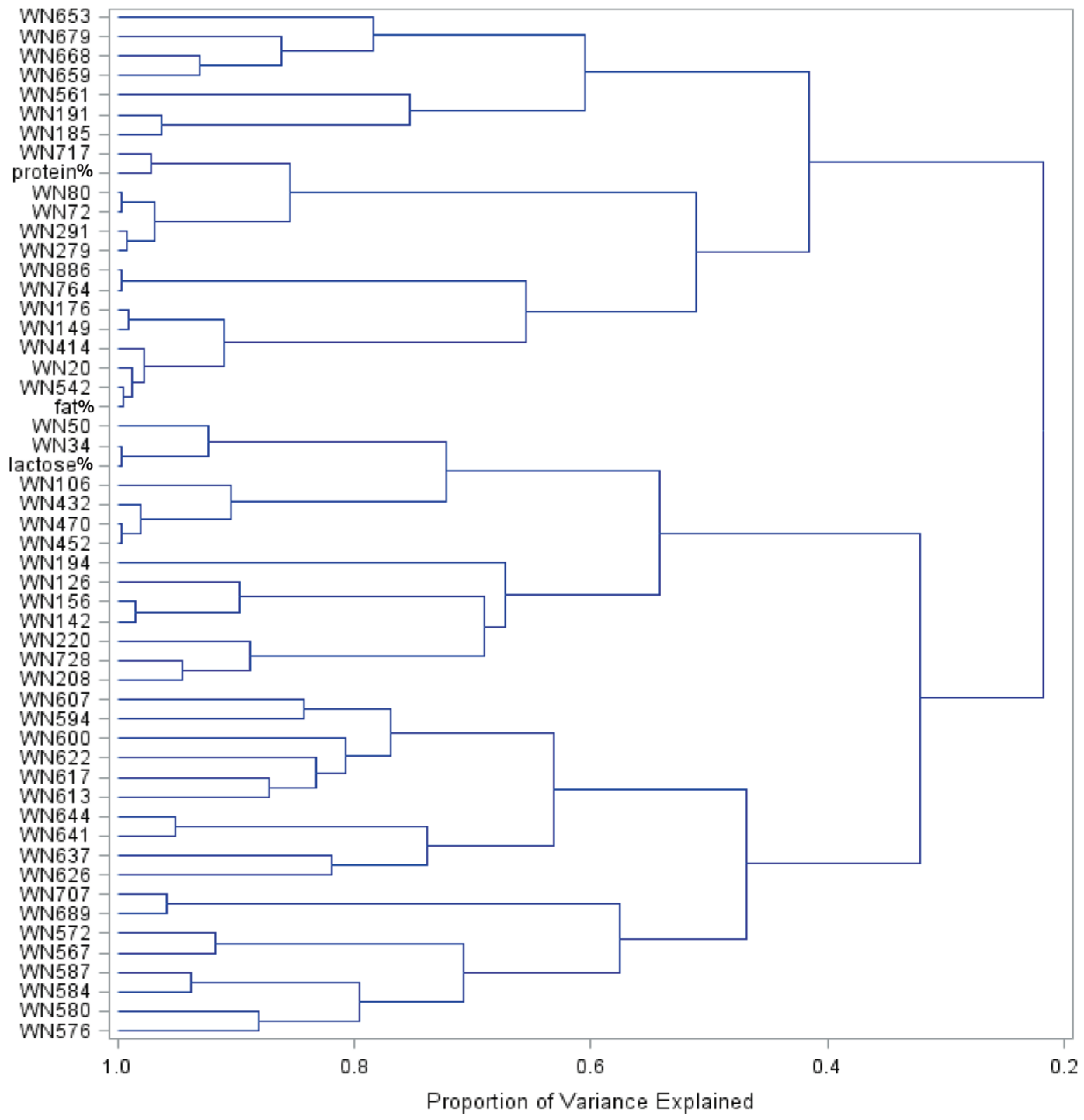

Figure 1. Cluster tree based on principal component analysis of phenotypic correlations among the infrared wavenumbers (WN) and fat, protein, and lactose percentage analyzed in this study. Color version available online. 

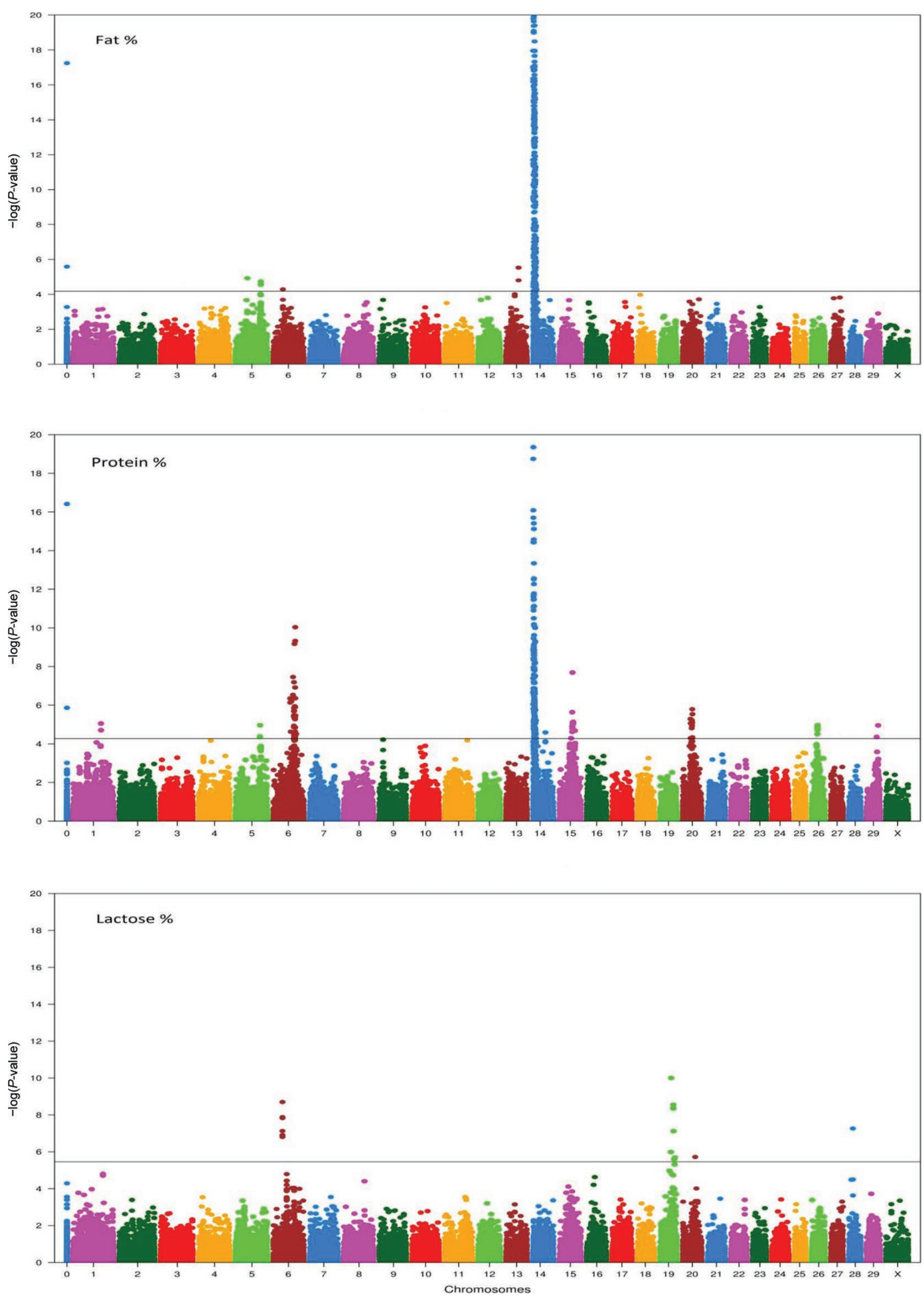

Figure 2. Manhattan plots of genome-wide association studies on infrared-predicted fat, protein, and lactose percentage. The genomic position is represented along the $\mathrm{x}$-axis and chromosome numbers are given. The $-\log _{10}(P)$ values of SNP are given on the $\mathrm{y}$-axis and cut off at 20 . The horizontal lines represent the 0.01 false-discovery rate thresholds. Color version available online. 
cant effects on multiple IR wavenumbers (e.g., WN72, WN80, WN149, WN176, WN717) with $-\log _{10}(P)$ ranging from 4.2 to 5.1, and on fat percentage with $-\log _{10}(P)$ of 4.9. Figure 1 shows that wavenumbers associated with region 5_a are phenotypically correlated with protein and fat percentage; however, protein percentage didn't show any association in this region. This region has not been associated with milk production traits in other studies.
In region 5_b, SNP were associated with WN72, WN80, WN176, WN279, WN717 [- $\log _{10}(P)$ up to 5.5], and fat percentage $\left[-\log _{10}(P)\right.$ up to 4.7]. The lead SNP rs29016908 was located at $101.1 \mathrm{Mbp}$. Region 5_b also contained several SNP (lead SNP rs41569048) significantly associated with protein percentage $\left[-\log _{10}(P)\right.$ up to 4.9]. The same region was previously reported by Schopen et al. (2011). Based on sequence-based imputation and expression QTL mapping, Littlejohn et al.
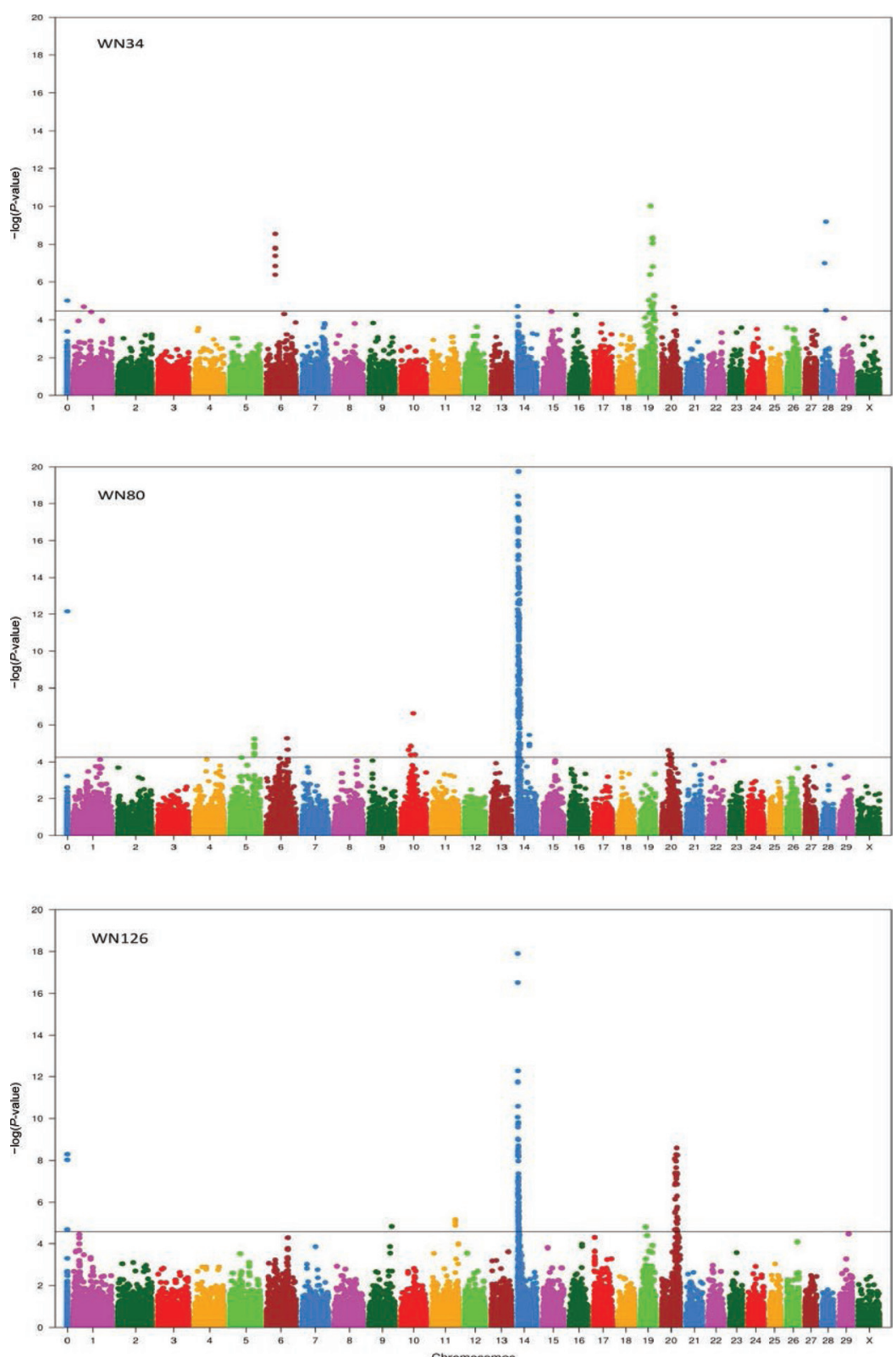

Figure 3. Manhattan plots of genome-wide association studies on representative infrared wavenumbers, showing all the significant genomic regions. The genomic position is represented along the $\mathrm{x}$-axis and chromosome numbers are given. The $-\log _{10}(P)$ values of SNP are given on the $\mathrm{y}$-axis and cut off at 20 . The horizontal lines represent the 0.01 false-discovery rate thresholds. Color version available online. 

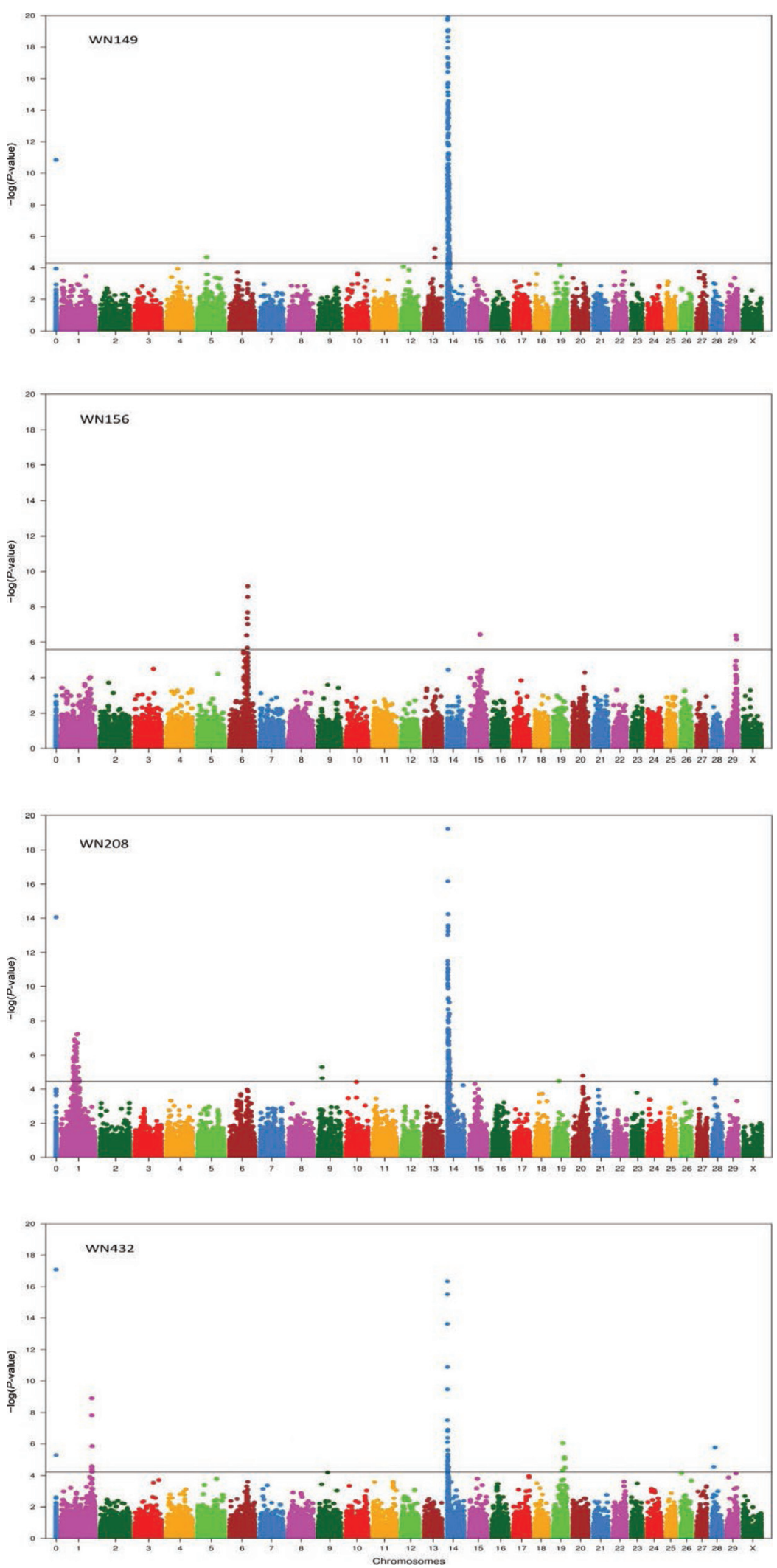

Figure 3 (Continued). Manhattan plots of genome-wide association studies on representative infrared wavenumbers, showing all the significant genomic regions. The genomic position is represented along the $\mathrm{x}$-axis and chromosome numbers are given. The $-\log _{10}(P)$ values of $\mathrm{SNP}$ are given on the y-axis and cut off at 20 . The horizontal lines represent the 0.01 false-discovery rate thresholds. Color version available online. 
(2016) identified microsomal glutathione S-transferase 1 (MGST1) as the most likely causal gene for the observed associations with fat percentage on BTA 5. Gene MGST1 is located at $93.5 \mathrm{Mbp}$, and Littlejohn et al. (2016) found this chromosomal region to be strongly associated with milk fat and protein percentage and milk yield.

\section{BTA 6}

We detected 2 significant regions on BTA 6: region 6_a, near 37.1 Mbp (see Figure 2 for lactose percentage and Figure 3 for WN34), and region 6_b, from 68.0 to $93.5 \mathrm{Mbp}$ (see Figure 2 for protein percentage and Figure 3 for WN156). Region 6_a was associated with WN34, WN50, WN106, WN452, WN470, WN728, and lactose percentage. For these wavenumbers the lead SNP rs81154100 showed $-\log _{10}(P)$ values ranging from 4.5 to 8.5. The association detected for region 6 _a with lactose percentage is in line with Kemper et al. (2016). As shown in Figure 1, several of the wavenumbers associated with region 6_a are strongly correlated with lactose percentage. Significant associations for this region have also been reported for SCS (Daetwyler et al., 2008), which is known to be negatively correlated with lactose percentage (e.g., Welper and Freeman, 1992).

Region 6_a harbors the gene ATP-binding cassette, subfamily $\mathrm{G}$, member 2 ( $A B C G 2$, located at $37.4 \mathrm{Mbp}$ ), which has been suggested to be the causative gene underlying the QTL (Cohen-Zinder et al., 2005; Olsen et al., 2007). The expression of ABCG2 in the mammary gland is induced during late pregnancy and the lactation period, contributing to the secretion of nutrients into the milk (Jonker et al., 2005). For instance, a major role for $A B C G 2$ in the secretion of antimicrobials and riboflavin into the milk of ruminants has been reported (Real et al., 2011; Otero et al., 2016).

In region 6_b, many SNP were very significantly associated with WN142, WN156, WN717, WN728, and WN764. For these wavenumbers, the lead SNP rs43703016 showed $-\log _{10}(P)$ values ranging from 7.9 to 17.6 , and for protein percentage the $-\log _{10}(P)$ value was 10.0. As shown in Figure 1, WN142, WN156, and WN728 were phenotypically strongly correlated, and WN717 was strongly correlated with protein percentage.

This region contains the 4 casein-coding genes. The lead SNP rs43703016 located at $88.5 \mathrm{Mbp}$ was the most significant SNP associated with these traits in the region. This SNP is 1 of $2 \mathrm{SNP}$ that are causal for protein variants $\mathrm{A}$ and $\mathrm{B}$ of $\kappa-\mathrm{CN}$. Schopen et al. (2011) showed that this SNP is strongly associated with protein percentage, $\beta$-LG content, casein index, and especially $\kappa$-CN content. Previously we detected significant effects of $\kappa$-CN milk protein variants on WN142, WN156, WN717, WN728, and WN764 (Wang et al., 2016).

\section{BTA 14}

We detected 2 significant regions on BTA 14: region 14_a, from 0 to $18.5 \mathrm{Mbp}$ (see Figure 2 for fat and protein percentage and Figure 3 for WN80, WN126, WN149, WN208, and WN432), and region 14_b, near 49.3 Mbp (see Figure 3 for WN80). Region 14_a was strongly associated with 21 of the 50 selected IR wavenumbers, as well as protein and fat percentage; WN279 showed the highest $-\log _{10}(P)$ of 103.4. Region 14_a contains the diacylglycerol O-acyltransferase 1 (DGAT1) gene. Previously, Wang et al. (2016) showed that the DGAT1 K232A polymorphism has significant effects on many milk IR wavenumbers. It has been shown in several studies that the DGAT1 K232A polymorphism has major effects on milk yield and milk fat and protein percentage (Grisart et al., 2002). Furthermore, Bovenhuis et al. (2016) showed that the DGAT1 K232A polymorphism has significant effects on milk fatty acid, protein, and mineral composition. We also found the significant association of region 14_a for WN668, which is part of the water absorption region. This agrees with our previous finding that the DGAT1 K232A polymorphisms are significantly associated with wavenumbers in the water absorption region (Wang et al., 2016). In this previous study, we found that wavenumbers from 3,466 to $3,543 \mathrm{~cm}^{-1}$ (including WN668) were significantly affected by the DGAT1 K232A polymorphism, with the highest $-\log _{10}(P)$ value of 17.0. The signals for wavenumbers in the so-called water absorption region are dominated by the effect of water in milk, but not completely. This suggests that these wavenumbers contain useful information regarding milk composition.

Remarkably, we detected another region on BTA 14 (14_b) affecting milk composition. Region 14_b was significantly associated with WN80 and WN142. Multiple SNP in this region (e.g., lead SNP rs41668861), which are located at approximately $49.3 \mathrm{Mbp}$, were significantly associated with WN80 $\left[-\log _{10}(P)\right.$ of 5.4 ; Figure 3] and WN142 $\left[-\log _{10}(P)\right.$ of 4.8]. The SNP in this region were also associated with protein percentage $\left[-\log _{10}(P)\right.$ of 4.6 ; Figure 2]. The SNP in this region remained significant after correcting for the DGAT1 K232A polymorphisms, suggesting that this is the effect of a different QTL. Bennewitz et al. (2004) suggested that, besides the DGAT1 K232A polymorphism, other QTL on BTA 14 affect milk production traits. Other studies reported a QTL in the region between 55 
and $79 \mathrm{Mbp}$ affecting milk production traits (Ashwell et al., 2004; Kolbehdari et al., 2009).

\section{BTA 19}

We detected significant associations for the region from 42.2 to $59.0 \mathrm{Mbp}$ on BTA 19. The associations for this region are illustrated for lactose percentage in Figure 2 and for WN34 and WN432 in Figure 3. The lead SNP, rs29020588, was significantly associated with WN20, WN34, WN50, WN106, WN432, WN452, and WN470, with $-\log _{10}(P)$ ranging from 4.6 to 10.0, and lactose percentage $\left[-\log _{10}(P)\right.$ of 10.0]. Another highly significant SNP was rs109400579 located at 52.0 Mbp and showed in general slightly lower $-\log _{10}(P)$ for the wavenumbers as compared with SNP rs29020588. Cecchinato et al. (2014) found a significant association for lactose percentage in Brown Swiss at $48.8 \mathrm{Mbp}$ and suggested that the growth hormone 1 gene (GH1) is involved.

As shown in Figure 1, all the wavenumbers associated with this region on BTA 19, except WN20, are strongly correlated. Bouwman et al. (2011) reported that BTA 19 is strongly associated with short- and mediumchain SFA (e.g., C14:0) and with long-chain UFA (e.g., C18:1). Bouwman et al. (2014) fine-mapped the region on BTA 19 associated with fatty acids and showed that the most significant SNP were located in a linkage disequilibrium block that contained the genes fatty acid synthase $(F A S N)$ and coiled-coil domain containing 57 $\left(C C D C 5^{\circ}\right)$. Gene $F A S N$ is involved in de novo fatty acid synthesis and has been associated with fat percentage and medium- and long-chain fatty acid content of milk (Roy et al., 2006; Morris et al., 2007). Medrano et al. (2010) showed that CCDC57 is expressed in the mammary gland, but this gene has not been related to milk fat composition. Another gene, ATP citrate lyase $(A C L Y)$, located at $42.7 \mathrm{Mbp}$, is a critical enzymelinking glucose catabolism to lipogenesis by providing acetyl-CoA from mitochondrial citrate for fatty acid and cholesterol biosynthesis. Some other genes located in this region are signal transducer and activator of transcription 5A (STAT5A) and sterol regulatory element binding transcription factor 1 (SREBF1). These genes are possibly related to fat composition or lactose percentage. Our study did not detect association for fat percentage in this region, which indicates that the QTL is responsible for relative amount of fatty acids but not total milk fat content.

\section{BTA 20}

We detected 2 significant regions on BTA 20: region 20_a, from 27.3 to $39.1 \mathrm{Mbp}$ (see Figure 2 for protein percentage and Figure 3 for WN20, WN80, WN142, WN279, WN717), and region 20_b, from 52.8 to $70.7 \mathrm{Mbp}$ (see Figure 3 for WN126). The lead SNP (rs41257066) for region 20_a showed $-\log _{10}(P)$ ranging from 4.9 to 5.8. This region harbors the growth hormone receptor $(G H R)$ gene, which has been reported to be associated with milk yield and composition (e.g., Blott et al., 2003, Kadri et al., 2015). Region 20_b is associated with WN106, WN176, and especially WN126 $\left[-\log _{10}(P)\right.$ of 8.6]. Another SNP in this region was also associated with lactose percentage (Figure 2); however, this SNP was not associated with WN126. Therefore, the highly significant association detected for WN126 was not detected for the routinely recorded milk composition traits fat, protein, and lactose percentage. For the same genomic region on BTA 20, Buitenhuis et al. (2013) reported a significant association for citric acid in Danish Holstein; therefore, WN126 might be related to citric acid. Recently, Grelet et al. (2016) showed that citrate in milk can be predicted with good accuracy based on milk IR data.

\section{Additional Genomic Regions}

BTA 10. A SNP located at $51.6 \mathrm{Mbp}$ was significantly associated with WN50, WN72, WN80, WN142, WN279, WN452, and WN717 with $-\log _{10}(P)$ ranging from 4.8 to 6.6. The association of this region is shown for WN80 in Figure 3. In Danish studies, this region on BTA 10 has been associated with milk fat composition and glycosylated $\kappa$-CN content (Buitenhuis et al., 2014, 2016). However, based on largely the same animals as used in the current study, Bouwman et al. (2012) did not detect significant associations of this region with milk fat composition.

BTA 11. The SNP located at approximately $95 \mathrm{Mbp}$ (e.g., lead SNP rs29014608) were significantly associated with WN126 $\left[-\log _{10}(P)\right.$ up to 5.2]. The association for this region is shown in Figure 3 for WN126. Wang et al. (2016) showed that the $\beta$-LG protein polymorphism has a significant effect on WN126. Schopen et al. (2011) reported that the region from 84.3 to $110.2 \mathrm{Mbp}$ is associated with milk protein composition, and SNP rs41255679 located at $107.2 \mathrm{Mbp}$ is associated with $\beta-\mathrm{LG}$ content and the casein index. This SNP is located in the promoter region of the $\beta-\mathrm{LG}$ gene and is known to be in linkage disequilibrium with variants $\mathrm{A}$ and $\mathrm{B}$ of $\beta$-LG (Ganai et al., 2009). Bedere and Bovenhuis (2017) showed that the tail part of BTA 11 contains more than 1 mutation with an effect on $\beta$-LG content.

BTA 13. Several SNP on BTA 13 (e.g., lead SNP rs41658332) located from 50.6 to $50.8 \mathrm{Mbp}$ were significantly associated with WN20, WN149, WN176, WN542, WN764, WN886 $\left[-\log _{10}(P)\right.$ ranging from 4.2 
to 5.8], and fat percentage $\left[-\log _{10}(P)\right.$ up to 5.5]. Figure 1 shows that these wavenumbers are correlated with each other and with fat percentage. The associations detected for this region are illustrated in Figure 2 for fat percentage and in Figure 3 for WN149. Bouwman et al. (2011) found a SNP located at $64.8 \mathrm{Mbp}$, which is in the gene acyl-CoA synthetase short-chain family member 2 (ACSS2). This is a candidate gene for C6:0, C8:0, and C10:0. Cole et al. (2011) reported several SNP located between 50 and $60 \mathrm{Mbp}$ are related to fat yield and fat percentage.

BTA 15. In the region around $51.9 \mathrm{Mbp}$, several SNP were significantly associated with WN142, WN156, and protein percentage, with $-\log _{10}(P)$ ranging from 5.5 to 7.7; SNP rs110249976 showed the most significant associations with these traits. This SNP was also reported by Schopen et al. (2011) to be significantly associated with protein percentage. The associations of this region were shown in Figure 2 for protein percentage and in Figure 3 for WN156.

BTA 28. The SNP rs29016491 at $14.9 \mathrm{Mbp}$ was significantly associated with WN34, WN50, WN106, WN208, WN432, WN470 $\left[-\log _{10}(P)\right.$ ranging from 4.7 to 9.2$]$, and lactose percentage $\left[-\log _{10}(P)\right.$ up to 7.3$\left.)\right]$ Another SNP at $8.7 \mathrm{Mbp}$ also showed significant associations with these wavenumbers. The associations of this region were shown in Figure 2 for lactose percentage and in Figure 3 for WN34. Most of these wavenumbers were associated with absorption bands of $\mathrm{C}-\mathrm{O}$ and $\mathrm{C}-\mathrm{H}$ bonds (Table 1), which are abundant in lactose.

BTA 29. The significant associations were detected at $45.4 \mathrm{Mbp}$ for WN156 and protein percentage; SNP rs29026584 was the most significant SNP affecting WN156 $\left[-\log _{10}(P)\right.$ of 6.2$]$ and protein percentage [$\log _{10}(P)$ of 5.0]. This SNP was also reported by Schopen et al. (2011) to be significantly associated with protein percentage, and Pryce et al. (2010) also found this region to be associated with protein percentage. Wavenumber 156 is related to the band of N-H stretching vibration; therefore, this genomic region might contain information on milk protein content. The associations for this region are illustrated in Figure 2 for protein percentage and in Figure 3 for WN156.

In the current study, we presented GWAS results for milk IR wavenumbers. Most of the identified genomic regions have been previously associated with fat, protein, or lactose percentage. Interestingly, several regions were also identified that could not be related to these routinely recorded traits. For instance, genomic regions were identified that in other studies have been associated with phosphorus, orotic acid, and citric acid. This suggests genetic variation in these milk components and that the milk IR spectra contain information about these milk components. Phosphorus, orotic acid, and citric acid are components with low concentrations in milk. Detection of genomic regions based on IR wavenumbers for these low-content components suggest that it might be of interest to calibrate IR for other low-content components. Large-scale recording of milk phosphorus content based on IR prediction equations can contribute to improved phosphorus efficiency of the dairy sector, whereas there is currently no clear application for large-scale recording of orotic or citric acid.

\section{CONCLUSIONS}

A GWAS was performed for 50 individual milk IR wavenumbers, resulting in 24 significant genomic regions on 16 bovine chromosomes. Out of the 50 individual milk IR wavenumbers, 28 wavenumbers showed significant associations with at least 1 genomic region. Genomic regions on chromosome 5, 6, 14, 19, and 20 showed significant associations with milk IR wavenumbers as well as milk composition traits, such as fat, protein, or lactose percentage. Remarkably, chromosome 1 contains 2 regions associated with milk IR wavenumbers but did not show an association with routinely recorded milk composition traits fat, protein, or lactose percentage. One of these regions has been shown to be associated with orotic acid and the other with phosphorus content of milk. This suggests that milk IR data contains information about phosphorus and orotic acid content of milk. The current study shows that GWAS based on milk IR wavenumbers revealed new genomic regions affecting milk composition that were not identified based on routinely recorded milk composition traits. Furthermore, this study shows the GWAS provides further insight in the information that is captured by the milk IR spectra. This can lead to novel applications of milk IR spectroscopy for dairy cattle breeding and herd management.

\section{ACKNOWLEDGMENTS}

The China Scholarship Council is acknowledged for funding the PhD project of Qiuyu Wang. Cooperative Cattle Improvement Organization (CRV, Arnhem, the Netherlands) is acknowledged for the sampling cows providing and imputation of genotypes. Milk Control Station (Zutphen, the Netherlands) is acknowledged for infrared spectra data. This study is part of the Dutch Milk Genomics Initiative and the project "Melk op Maat," funded by Wageningen University (Wageningen, the Netherlands), the Dutch Dairy Association (NZO, Zoetermeer, the Netherlands), CRV, the Dutch Technology Foundation (STW, Utrecht, the Netherlands), 
the Dutch Ministry of Economic Affairs (The Hague, the Netherlands), and the Provinces of Gelderland and Overijssel (Arnhem, the Netherlands).

\section{REFERENCES}

Ashwell, M. S., D. W. Heyen, T. S. Sonstegard, C. P. Van Tassell, Y. Da, P. M. VanRaden, M. Ron, J. I. Weller, and H. A. Lewin. 2004. Detection of quantitative trait loci affecting milk production, health, and reproductive traits in Holstein cattle. J. Dairy Sci. 87:468-475.

Bedere, N., and H. Bovenhuis. 2017. Characterizing a region on BTA11 affecting $\beta$-lactoglobulin content of milk using high-density genotyping and haplotype grouping. BMC Genet. 18:17.

Bennewitz, J., N. Reinsch, S. Paul, C. Looft, B. Kaupe, C. Weimann, G. Erhardt, G. Thaller, C. Kühn, M. Schwerin, and H. Thomsen. 2004. The DGAT1 K232A mutation is not solely responsible for the milk production quantitative trait locus on the bovine chromosome 14. J. Dairy Sci. 87:431-442.

Bittante, G., and A. Cecchinato. 2013. Genetic analysis of the Fouriertransform infrared spectra of bovine milk with emphasis on individual wavenumbers related to specific chemical bonds. J. Dairy Sci. 96:5991-6006.

Blott, S., J. J. Kim, S. Moisio, A. Schmidt-Kuntzel, A. Cornet, P. Berzi, N. Cambisano, C. Ford, B. Grisart, and D. Johnson. 2003. Molecular dissection of a quantitative trait locus: A phenylalanineto- tyrosine substitution in the transmembrane domain of the bovine growth hormone receptor is associated with a major effect on milk yield and composition. Genetics 163:253-266.

Bonfatti, V., L. Degano, A. Menegoz, and P. Carnier. 2016. Mid-infrared spectroscopy prediction of fine milk composition and technological properties in Italian Simmental. J. Dairy Sci. 99:8216-8221.

Bouwman, A. C., H. Bovenhuis, M. H. Visker, and J. M. van Arendonk. 2011. Genome-wide association of milk fatty acids in Dutch dairy cattle. BMC Genet. 12:43.

Bouwman, A. C., M. H. Visker, J. M. van Arendonk, and H. Bovenhuis. 2012. Genomic regions associated with bovine milk fatty acids in both summer and winter milk samples. BMC Genet. 13:93.

Bouwman, A. C., M. H. Visker, J. M. van Arendonk, and H. Bovenhuis. 2014. Fine mapping of a quantitative trait locus for bovine milk fat composition on Bos taurus autosome 19. J. Dairy Sci. 97:1139-1149.

Bovenhuis, H., M. H. P. W. Visker, N. A. Poulsen, J. Sehested, H. J F. van Valenberg, J. A. M. van Arendonk, L. B. Larsen, and A. J. Buitenhuis. 2016. Effects of the diacylglycerol o-acyltransferase 1 (DGAT1) K232A polymorphism on fatty acid, protein, and mineral composition of dairy cattle milk. J. Dairy Sci. 99:3113-3123.

Buitenhuis, A. J., U. K. Sundekilde, N. A. Poulsen, H. C. Bertram, L. B. Larsen, and P. Sørensen. 2013. Estimation of genetic parameters and detection of quantitative trait loci for metabolites in Danish Holstein milk. J. Dairy Sci. 96:3285-3295.

Buitenhuis, B., L. L. Janss, N. A. Poulsen, L. B. Larsen, M. K. Larsen, and P. Sørensen. 2014. Genome-wide association and biological pathway analysis for milk-fat composition in Danish Holstein and Danish Jersey cattle. BMC Genomics 15:1112.

Buitenhuis, B., N. A. Poulsen, G. Gebreyesus, and L. B. Larsen. 2016. Estimation of genetic parameters and detection of chromosomal regions affecting the major milk proteins and their post translational modifications in Danish Holstein and Danish Jersey cattle. BMC Genet. 17:114.

Buitenhuis, B., N. A. Poulsen, L. B. Larsen, and J. Sehested. 2015. Estimation of genetic parameters and detection of quantitative trait loci for minerals in Danish Holstein and Danish Jersey milk. BMC Genet. 16:52.

Cecchinato, A., C. Ribeca, S. Chessa, C. Cipolat-Gotet, F. Maretto, J. Casellas, and G. Bittante. 2014. Candidate gene association analysis for milk yield, composition, urea nitrogen and somatic cell scores in Brown Swiss cows. Animal 8:1062-1070.
Chou, J. Y., H. S. Jun, and B. C. Mansfield. 2013. The SLC37 family of phosphate-linked sugar phosphate antiporters. Mol. Aspects Med. 34:601-611.

Cohen-Zinder, M., E. Seroussi, D. M. Larkin, J. J. Loor, A. Everts-van der Wind, J. H. Lee, J. K. Drackley, M. R. Band, A. G. Hernandez, M. Shani, and H. A. Lewin. 2005. Identification of a missense mutation in the bovine ABCG2 gene with a major effect on the QTL on chromosome 6 affecting milk yield and composition in Holstein cattle. Genome Res. 15:936-944.

Cole, J. B., G. R. Wiggans, L. Ma, T. S. Sonstegard, T. J. Lawlor, B. A. Crooker, C. P. van Tassell, J. Yang, S. Wang, L. K. Matukumalli, and Y. Da. 2011. Genome-wide association analysis of thirty one production, health, reproduction and body conformation traits in contemporary US Holstein cows. BMC Genomics 12:408.

Daetwyler, H. D., F. S. Schenkel, M. Sargolzaei, and J. A. B. Robinson. 2008. A genome scan to detect quantitative trait loci for economically important traits in Holstein cattle using two methods and a dense single nucleotide polymorphism map. J. Dairy Sci. 91:3225-3236.

Dehareng, F., C. Delfosse, E. Froidmont, H. Soyeurt, C. Martin, N. Gengler, A. Vanlierde, and P. Dardenne. 2012. Potential use of milk mid-infrared spectra to predict individual methane emission of dairy cows. Animal 6:1694-1701.

Diem, M. 2015. Modern Vibrational Spectroscopy and Micro-Spectroscopy: Theory, Instrumentation and Biomedical Applications. John Wiley \& Sons, Hoboken, NJ.

Ganai, N. A., H. Bovenhuis, J. A. M. van Arendonk, and M. H. P. W. Visker. 2009. Novel polymorphisms in the bovine $\beta$-lactoglobulin gene and their effects on $\beta$-lactoglobulin protein concentration in milk. Anim. Genet. 40:127-133.

Gilmour, A. R., B. J. Gogel, B. R. Cullis, and R. Thompson. 2009. ASReml User Guide Release 3.0. VSN International Ltd., Hemel Hempstead, UK.

Grelet, C., C. Bastin, M. Gelé, J.-B. Davière, M. Johan, A. Werner, R. Reding, J. A. Fernandez Pierna, F. G. Colinet, P. Dardenne, N. Gengler, H. Soyeurt, and F. Dehareng. 2016. Development of Fourier transform mid-infrared calibrations to predict acetone, $\beta$-hydroxybutyrate, and citrate contents in bovine milk through a European dairy network. J. Dairy Sci. 99:4816-4825.

Grisart, B., W. Coppieters, F. Farnir, L. Karim, C. Ford, P. Berzi, N. Cambisano, M. Mni, S. Reid, P. Simon, R. Spelman, M. Georges, and R. Snell. 2002. Positional candidate cloning of a QTL in Dairy cattle: Identification of a missense mutation in the bovine DGAT1 gene with major effect on milk yield and composition. Genome Res. 12:222-231.

ICAR (International Committee for Animal Recording). 2012. International Agreement of Recording Practices - Guidelines Approved by the GENERAL ASSEMBLY held in Cork, Ireland on June 2012. ICAR, Rome. Italy.

Jonker, J. W., G. Merino, S. Musters, A. E. van Herwaarden, E. Bolscher, E. Wagenaar, E. Mesman, T. C. Dale, and A. H. Schinkel. 2005. The breast cancer resistance protein BCRP (ABCG2) concentrates drugs and carcinogenic xenotoxins into milk. Nat. Med. 11:129.

Kadri, N. K., B. Guldbrandtsen, S. M. Lund, and G. Sahana. 2015. Genetic dissection of milk yield traits and mastitis resistance quantitative trait loci on chromosome 20 in dairy cattle. J. Dairy Sci. 98:9015-9025.

Kemper, K. E., M. D. Littlejohn, T. Lopdell, B. J. Hayes, L. E. Bennett, R. P. Williams, X. Q. Xu, P. M. Visscher, M. J. Carrick, and M. E. Goddard. 2016. Leveraging genetically simple traits to identify small-effect variants for complex phenotypes. BMC Genomics 17:858.

Kolbehdari, D., Z. Wang, J. R. Grant, B. Murdoch, A. Prasad, Z. Xiu, E. Marques, P. Stothard, and S. S. Moore. 2009. A whole genome scan to map QTL for milk production traits and somatic cell score in Canadian Holstein bulls. J. Anim. Breed. Genet. 126:216-227.

Littlejohn, M. D., K. Tiplady, T. A. Fink, K. Lehnert, T. Lopdell, T. Johnson, C. Couldrey, M. Keehan, R. G. Sherlock, C. Harland, A. Scott, R. G. Snell, S. R. Davis, and R. J. Spelman. 2016. Sequence- 
based association analysis reveals an MGST1 eQTL with pleiotropic effects on bovine milk composition. Sci. Rep. 6:25376.

Liu, Y., X. Qin, X. Z. H. Song, H. Jiang, Y. Shen, K. J. Durbin, S. Lien, M. P. Kent, M. Sodeland, Y. Ren, L. Zhang, E. Sodergren, P. Havlak, K. C. Worley, G. M. Weinstock, and R. A. Gibbs. 2009. Bos taurus genome assembly. BMC Genomics 10:180.

McParland, S., G. Banos, E. Wall, M. P. Coffey, H. Soyeurt, R. F. Veerkamp, and D. P. Berry. 2011. The use of mid-infrared spectrometry to predict body energy status of Holstein cows. J. Dairy Sci. 94:3651-3661.

Medrano, J., G. Rincon, and A. Islas-Trejo. 2010. Comparative analysis of bovine milk and mammary gland transcriptome using RNASeq. Page 125 in Proc. 9th World Congr. Genet. Appl. Livest. Prod., Leipzig, Germany. German Society for Animal Science, Neustadt, Germany.

Morris, C. A., N. G. Cullen, B. C. Glass, D. L. Hyndman, T. R. Manley, S. M. Hickey, J. C. McEwan, W. S. Pitchford, C. D. Bottema, and M. A. Lee. 2007. Fatty acid synthase effects on bovine adipose fat and milk fat. Mamm. Genome 18:64-74.

Olsen, H. G., H. Nilsen, B. Hayes, P. R. Berg, M. Svendsen, S. Lien, and T. Meuwissen. 2007. Genetic support for a quantitative trait nucleotide in the ABCG2 gene affecting milk composition of dairy cattle. BMC Genet. 8:32.

Otero, J. A., V. Miguel, L. González-Lobato, R. García-Villalba, J. C. Espín, J. G. Prieto, G. Merino, and A. I. Alvarez. 2016. Effect of bovine ABCG2 polymorphism Y581S SNP on secretion into milk of enterolactone, riboflavin and uric acid. Animal 10:238-247.

Pryce, J. E., S. Bolormaa, A. J. Chamberlain, P. J. Bowman, K. Savin, M. E. Goddard, and B. J. Hayes. 2010. A validated genome-wide association study in 2 dairy cattle breeds for milk production and fertility traits using variable length haplotypes. J. Dairy Sci. 93:3331-3345.

R Core Team. 2013. R: A language and environment for statistical computing. R Foundation for Statistical Computing, Vienna, Austria. http://www.R-project.org/.

Real, R., L. González-Lobato, M. F. Baro, S. Valbuena, A. de la Fuente, J. G. Prieto, A. I. Álvarez, M. M. Marques, and G. Merino. 2011. Analysis of the effect of the bovine adenosine triphosphate-binding cassette transporter G2 single nucleotide polymorphism Y581S on transcellular transport of veterinary drugs using new cell culture models. J. Anim. Sci. 89:4325-4338.

Robinson, J. L., D. B. Dombrowski, G. W. Harpestad, and R. D. Shanks. 1984. Detection and prevalence of UMP synthase deficiency among dairy cattle. J. Hered. 75:277-280.
Roy, R., L. Ordovas, P. Zaragoza, A. Romero, C. Moreno, J. Altarriba, and C. Rodellar. 2006. Association of polymorphisms in the bovine FASN gene with milk-fat content. Anim. Genet. 37:215-218.

Rutten, M. J. M., H. Bovenhuis, K. A. Hettinga, H. J. F. van Valenberg, and J. A. M. van Arendonk. 2009. Predicting bovine milk fat composition using infrared spectroscopy based on milk samples collected in winter and summer. J. Dairy Sci. 92:6202-6209.

Sanchez, M. P., A. Govignon-Gion, M. Ferrand, M. Gelé, D. Pourchet, Y. Amigues, S. Fritz, M. Boussaha, A. Capitan, D. Rocha, G. Miranda, P. Martin, M. Brochard, and D. Boichard. 2016. Wholegenome scan to detect quantitative trait loci associated with milk protein composition in 3 French dairy cattle breeds. J. Dairy Sci 99:8203-8215.

SAS Institute. 2011. SAS/STAT User's Guide: Release 9.3. SAS Inst., Cary, NC.

Schopen, G. C. B., M. H. P. W. Visker, P. D. Koks, E. Mullaart, J. A. M. van Arendonk, and H. Bovenhuis. 2011. Whole-genome association study for milk protein composition in dairy cattle. J. Dairy Sci. 94:3148-3158.

Soyeurt, H., D. Bruwier, J. M. Romnee, N. Gengler, C. Bertozzi, D. Veselko, and P. Dardenne. 2009. Potential estimation of major mineral contents in cow milk using mid-infrared spectrometry. J. Dairy Sci. 92:2444-2454.

Soyeurt, H., P. Dardenne, F. Dehareng, G. Lognay, D. Veselko, M Marlier, C. Bertozzi, P. Mayeres, and N. Gengler. 2006. Estimating fatty acid content in cow milk using mid-infrared spectrometry. J. Dairy Sci. 89:3690-3695.

Soyeurt, H., I. Misztal, and N. Gengler. 2010. Genetic variability of milk components based on mid-infrared spectral data. J. Dairy Sci. 93:1722-1728.

Sun, D. W., ed. 2009. Pages 3-27 in Infrared Spectroscopy for Food Quality Analysis and Control. Academic Press/Elsevier, London, UK.

Toffanin, V., M. De Marchi, N. Lopez-Villalobos, and M. Cassandro. 2015. Effectiveness of mid-infrared spectroscopy for prediction of the contents of calcium and phosphorus, and titratable acidity of milk and their relationship with milk quality and coagulation properties. Int. Dairy J. 41:68-73.

Wang, Q., A. Hulzebosch, and H. Bovenhuis. 2016. Genetic and environmental variation in bovine milk infrared spectra. J. Dairy Sci. 99:6793-6803

Welper, R. D., and A. E. Freeman. 1992. Genetic parameters for yield traits of Holsteins, including lactose and somatic cell score. J. Dairy Sci. 75:1342-1348. 